\title{
Mind Monitoring via Mobile Brain-body Imaging
}

\author{
Scott Makeig ${ }^{1}$ \\ ${ }^{1}$ Swartz Center for Computational Neuroscience, Institute for Neural \\ Computation, University of California, San Diego, USA \\ scott@sccn.ucsd.edu
}

\begin{abstract}
Current brain-computer interface (BCI) research attempts to estimate intended operator body or cursor movements from his/her electroencephalographic (EEG) activity alone. More general methods of monitoring operator cognitive state, intentions, motivations, and reactions to events might be based on continuous monitoring of the operator's (EEG) as well as his of her body and eye movements and, to the extent possible, her or his multisensory input. Joint modeling of this data should attempt to identify individualized modes of brain/body activity and/or reactivity that appear in the operator's brain and/or behavior in distinct cognitive contexts, if successful producing, in effect, a new mobile brain/body imaging (MoBI) modality. Robust MoBI could allow development of new brain/body-system interface (BBI) designs performing multidimensional monitoring of an operator's changing cognitive state including their movement intentions and motivations and ('topdown') apprehension of sensory events.
\end{abstract}

Key words: cognitive monitoring; electroencephalography (EEG); motion capture; independent component analysis (ICA); brain-computer interface (BCI), mobile brain/body imaging (MoBI); human-computer interface (HCI)

\section{Introduction}

Over the last decade there has been an explosion of interest in using EEG to monitor selected movement intentions of an operator trained to produce changes in the amplitude of one or more EEG measures that are mechanically associated by a braincomputer interface (BCI) system with two or more intended external actions (in simplest form, moving a screen cursor up or down). BCI research was first funded to construct systems allowing communication by a relatively few cognitively intact but totally paralyzed or 'locked-in' subjects though, naturally, first exploratory phases of BCI research use normal test subjects. To insure the possibility that the methods developed in these phases might be usable by the target locked-in subjects, it was important to establish that the EEG changes used to detect movement intentions were not based on non-brain contributions to EEG signals recorded on the scalp, e.g., activity arising from subject eye movements or scalp muscle activities. Thus, for many researchers the BCI concept became identified with the goal of using 'pure' EEG, apart from non-brain 'artifacts,' to convey and decipher a subject's stereotyped cursor (or body) movement intentions. 
The goal of providing a useful, non-invasive communication system for 'lockedin' subjects is surely laudable, and actual demonstrations that both a few 'locked-in' and many normal subjects can communicate (albeit quite slowly) via learned control of their macroscopic brain activity patterns, without involvement of direct motor control, are novel and intriguing. However, unnecessary adherence to this limited BCI goal could slow development of more general classes of human-system interfaces involving continuous monitoring of non-invasively recorded brain activity.

\subsection{Unexplored problems in BCI research}

As a new subject, at least four fundamental questions about the operation, limitations, and effects of EEG-based BCI operation remain unexplored:

1. Key obstacles to widespread acceptance and application of non-invasive EEGbased BCI systems are the need for a long training regimen, and the failure of a significant fraction of subjects to achieve stable, non-invasive BCI control even after intensive training. Finding specific reasons for these difficulties, and methods around them, are fundamental if BCI or more general 'neurotechnology' or 'neuroergonomic' HCI research is to have broad applications.

2. When a subject in a BCI experiment learns to move a computer screen cursor by increasing or reducing the amplitude of a selected brain rhythm - whether a mu rhythm, near-DC potential, or other phenomenon - what 'body part' (or brain system) do they use to willfully effect the modulation? While this is a fundamental issue for BCI research, it is one that has so far been nearly ignored.

3. Although achieving volitional control of a BCI system through EEG modulation alone is an intriguing goal, more general questions for HCI systems involving EEG monitoring are how to combine EEG analysis with concurrent recording and analysis of subject behavior, eye and muscle activities, and multisensory input to monitor and adapt to changing human cognitive state, intent, and reactivity.

4. Another relatively unexplored question is whether there are psychobiological effects of training and performing volitional control of natural brain rhythms. These effects might either be phasic (affecting the operator only during BCI operation) or tonic (also affecting their behavior and/or brain activity at other times); they might be positive (for example producing a useful strengthening of attentional control), or negative (some unforeseen consequence of disrupting natural, non-conscious modes of dynamic brain regulation).

All these questions should and must eventually be addressed by the advancing fields of human neuroscience and neurotechnology. This paper discusses a general plan of approach to the first three questions above - How can learning of EEG-based volitional control be made quicker and more universal? What EEG modulatory systems do successful BCI subjects use to learn and to effect volitional control of their EEG activity? And, how can EEG be combined with other information about operator behavior and sensation to allow human-system interactions to estimate and use information about operator mental state and cognitive reactions to events? 


\subsection{EEG Modulation}

EEG dynamics have long been characterized by their diverse spectral profiles. For example, slow semi-rhythmic activity is characteristic of EEG in deep sleep, while awake/alert EEG contains more high-frequency activity. Narrow-band brain rhythms appear most predominantly in the $(8-12 \mathrm{~Hz})$ alpha band, but also at somewhat higher and lower frequencies. Spectral modulations of EEG activity at lower and higher frequencies affect broader frequency bands. A considerable (if insufficient) amount is known about several brain systems that modulate the spectrum of local field activity in the brain's cortex, the brain source of most scalp-recorded EEG. A number of these systems are the brainstem-centered 'evaluation' systems labeled by the specific neurotransmitter they project quite widely (acetylcholine, dopamine, norepinephrine, serotonin, or etc.). However, evidence for the involvement of these or other systems in successful BCI control has not been presented.

\subsection{Mobile brain/body imaging (MoBI)}

The fundamental purpose of the brain is to control behavior or more exactly, to optimize the outcome of behavior - maximizing its ensuing rewards and/or minimizing ensuing penalties as per subject purposes, needs, and desires. It is now possible to record brain activity at relatively high bandwidth - a Mbit/sec or more of EEG, MEG, BOLD, single-cell spike/field data, etc. Surprisingly, however, there has been little serious effort to concurrently record the behavior the brain is controlling with anything near the same bandwidth. In human brain experiments, behavior is most often recorded only in the form of a sparse series of minimal finger button presses - giving an effective rate of behavioral data collection near $1 \mathrm{bit} / \mathrm{sec}$. Simply from this $\sim 1,000,000: 1$ mismatch, it is no wonder that recent progress in human psychophysiology has been relatively slow.

The obvious remedy for this oversight is to simultaneously record as much behavioral information as possible in paradigms including some range of natural behavior. It should be desirable to record as wide and natural a range of behavior as possible, in as physically free and natural a behavioral environment as possible. Currently, this goal can only be approached only using EEG brain imaging, since its sensors, alone among current high-bandwidth brain imaging modalities, are light enough that its recording does not require major restriction on subject head or body movements.

Recently, I have proposed the combination of wearable, high-density EEG and body motion capture (combined, ideally, with eye gaze and audiovisual scene recording) may constitute a new brain imaging modality, 'Mobile Brain/Body Imaging' or MoBI [1]. Once successfully developed and demonstrated, MoBI could allow, for the first time, the study of macroscopic brain dynamic patterns supporting natural and naturally motivated actions (and interactions) in normal 3-D environments. 
A key first problem to be overcome in realizing the promise of mobile brain imaging is the problem of separating the activities of brain EEG sources from nonbrain artifacts, particularly head and neck muscle activities and artifacts induced in the EEG by eye movements. A workable solution to this problem, at least, is the introduction of independent component analysis (ICA) of EEG data [2-6]. Under favorable circumstances, ICA cleanly separates brain and non-brain data source activities that are mixed by volume conduction in scalp EEG recordings, a process for which much open-source software is now available [7]. A second problem is to model the muscular forces producing the observed motor behavior; for this, open-source biomechanical modeling software is also becoming available [8]. Finally, adequate statistical signal processing or machine learning methods are required to discover dynamic links between concurrent brain source activities, muscle activations, and other classes of MoBI data.

Supposing the near-future availability of viable MoBI recording and analysis methods, we can ask how the concept of BCI can be expanded to consider brain/body interface $(B B I)$ designs that acquire and continuously update information about the cognitive state, reactions, intentions, and motivations of the system operator from joint MoBI recording.

\section{Brain/body Interface (BBI) Methods}

For a BBI system to be maximally effective, it would seem wise to consider and test two design principles:

a) To best understand the complex associations of ongoing multidimensional changes in EEG dynamics with cognitive state, perceptual events, and movement intentions and motivations, the analysis should both observe and take into account the subject's movements (including limb, body, and eye movements), and any other available physiological signals. In other words, to optimally model brain activity it is important to take in to account, as much as possible, the behavior the brain is controlling. This suggests the potential importance of the development of concurrent brain/body imaging recording and analysis, as in the MoBI concept.

b) The information about cognitive state and action motivations and intentions that may be most robustly decoded from joint EEG and behavioral information should concern distinctions between circumstances and events in which EEG dynamics exhibit spontaneous differences. In particular, it is likely that learned control of EEG signals will be most successful when the learned repertoire of EEG modulations used to decode subject control intentions are identical or close to the subject's repertoire of spontaneous EEG modulations.

The identified EEG dynamics used in BBI monitoring and control may either index brain dynamics that play supporting roles in these circumstances, or their cortical source activities may also play a direct role in shaping the joint timing of 
distributed neural activities, a concept that is gradually being re-introduced into neuroscience by new evidence and by theoretical considerations of the utility of mass action in the central nervous system for controlling behavior and its outcomes.

\subsection{EEG Modulators}

Standard methods for analyzing EEG data are based on averaging measures of EEG dynamics across trials or time windows, thereby collapsing the continuously time-varying signals into a average representation of activity time-locked to one or more classes of events. Further, most EEG analyses focuses on the individual scalp channel signals, though they are each differently weighted mixtures of many brain and non-brain source signals. Independent component analysis attempts to locate discrete sources of information in multidimensional data in which several independent information streams are linearly mixed in sensor data. However, the spectrum of each identified brain source component signal, like every recorded scalp signal, varies irregularly over time. Standard methods for analyzing either independent component or scalp channel signals during a period of continued subject task performance typically model the exhibited variability as noisy deviations from a stable mean spectrum or stable event-related spectral perturbation (ERSP) time/frequency mean, variation noise in which spectral power at each frequency is implicitly assumed to vary independently.

An alternate approach assumes that the observed power spectral variability sums variations in several to many modes of spectral variability (and co-variability) that are characteristic of the component source process. Earlier, we introduced the device of converting component spectrograms to log power while positing that the motive force behind these modal modulations are processes that modulate spectral activity multiplicatively, at characteristic frequencies, with independent or near-independent time courses or effect distributions across trials [9]. Recently, we have tested the use of ICA decomposition the ongoing log power spectrograms of a number of independent component processes from single subjects performing eyes-closed imagination exercises ${ }^{1}$. Log spectral decomposition separated second-to-second variations in the $\log$ spectrogram into a $\log$ sum of multiplicative modulator processes, each with a fixed spectral and spatial component effect template whose effect on the affected spatial component log spectra is determined by multiplication by a single log amplitude time series. This approach gave a number of interesting results including alpha band processes at different frequencies plus harmonics, broader beta and theta band processes, and very broadband shifts in power distribution.

We have also experimented with adding information to the analysis about the time locking and other experimental events and the context in which they occur. The goal of this analysis approach is to avoid so far as possible the method of planned comparisons, the basis for most experimental data analysis, in which measures for

${ }^{1}$ Onton, J. and Makeig, S., unpublished data 
pairs of conditions are compared, each measure an identically weighted average of measurements characterized by one (or sometimes more) key variable value.

For example, there have been thousands of EEG studies that compared the average responses (typically called 'P300') to 'target' and 'non-target' stimuli in a simple attention task. The underlying assumption here is that the brain emits identical responses to each 'target' or 'non-target' stimulus, respectively, regardless of the local event context. The hope is that the effect of the 'target/non-target' variable is separable from other variables, and essentially stable across time. Unfortunately, this is not the case. P300 'target' responses vary widely in amplitude and scalp distribution from trial to trial, and this type of variability limits the performance of simple BCI systems, for example one that might attempt to set a fixed threshold to identify the appearance of a 'target' response, regardless of event context [10].

I propose that BBI research explore an alternative approach in which multiple characteristic relationships between EEG dynamics and single events in context are determined directly from the joint EEG, stimulus, and behavioral data. Some facts concerning the nature of individual events may be available to at BBI system in real time, for example the moment and screen on which a piece of information is presented, or the screen to which the subject is directing their gaze.

An example of an unavailable context variable might be the interpretation of the subject of a visual event as representing a challenge or threat. In pilot data recorded to build an individualized (or collective) BBI model, the level of threat could be varied systematically and the level of perceived threat might be estimated from the subject's brain and behavioral responses. In subsequent real-time operation, other variables defining the current event and event context may be available from the system event $\log$ and subject behavioral record.

Combined with direct observation of the EEG and subject motor behavior, these available context variables, combined, may allow estimation of the unavailable variable - here, whether and to what extent the subject perceives a visual event to signal a threat to the operation of the system. This information might be used to immediately deploy available additional countermeasures whenever a genuine perceived system threat is estimated to occur, or possibly to monitor the state of responsiveness of the subject when false indications of (test) threats are delivered to the subject, probing their advancing level of expertise in recognizing a threatening event, or for estimating their current cognitive fitness for duty.

If the system response to the operator's appraisal of a threatening event helps the operator mount an adequate and timely response, then the system response will serve as a powerful reward, and naturally over time and use the operator's EEG pattern should be expected to adapt to give a more distinct perceived-threat signal to the system. Thus, a natural cognitive response monitoring system could easily become an interactive learned $\mathrm{BCI} / \mathrm{BBI}$ system. Further, it is natural to hypothesize that when the system is based on the operator's natural brain response modes, it may also be natural 
and relatively easy for the operator to learn to produce the EEG patterns that are most distinctly and reliably detected by the system.

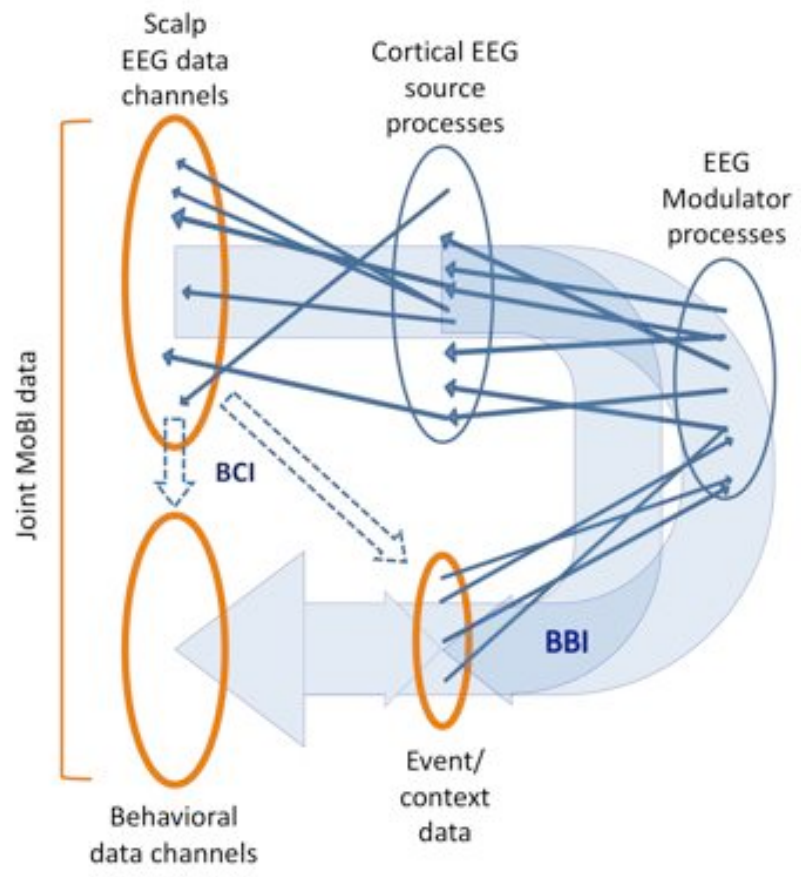

Fig. 1. Schematic model diagram for a non-invasive brain/behavior system interface (BBI) design. Concurrent scalp EEG, behavior, and event/context data are collected in a Mobile Brain/body Imaging (MoBI) paradigm (thick ovals). In most currently proposed BCI systems (dotted arrows), selected EEG data are processed in near-real time to estimate or predict some behavioral or event/context parameter (' $B C I$ '). In the proposed BBI, the EEG data are first separated into cortical EEG source processes (upper middle oval) (plus non-brain artifact processes, not shown). Then the time/frequency behaviors of the source processes are further separated into effects of a number of maximally distinct EEG source modulator processes (upper right oval). In the BBI model, both selected EEG time-domain source and frequencydomain source modulator data may be integrated with the behavioral and other event/context data to estimate or predict selected behavioral and/or event/context parameters (broad light blue arrows).

Fig. 1 gives the gist of the concept in graphic form. Three types of MoBI data may be recorded concurrently to run a brain/body interface (BBI): high-density EEG data, behavioral data, and context data (event information, event, EEG, and behavioral history, etc.). Standard BCI systems (dotted arrows) attempt to estimate some parameter of the behavioral and/or event/context data directly from the scalp EEG using a machine learning approach. In the proposed BBI model (wide light blue arrows), the EEG data are first separated into cortical (and non-brain) EEG source processes (thin blue arrows), the spectral modulator processes operating on these source processes are estimated from the EEG source data, and the linkage of the EEG source and source modulator processes to the behavioral and/or event/context data are 
determined. When one or more parameters of the event/context data are unavailable (e.g., in real-time operation), any of the available MoBI data may be used in a BBI to estimate the unavailable parameter. The estimation process might be designed to perform well even when additional data variables are missing. The MoBI data used for this estimation might include available behavioral data (body motion capture, eye gaze tracking, etc.) and event/context information as well as EEG dynamics.

\section{DISCUSSION}

The model of an EEG-based BBI system shown in Fig. 1 has the advantage of involving volitional control of spatiotemporal EEG dynamic patterns most specifically associated with the operator's spontaneous EEG responses in the targeted event categories [11]. While it is natural to hypothesize that strengthening and controlling spontaneously active EEG patterns may be more easily and quickly learned, this assumption may prove incorrect in some or many cases, and thus basic experiments (and adequate analyses) are needed to test it. Earlier, we showed that applying even highly overlearned BCI control of a single pre-defined EEG feature may involve complex and asymmetric EEG changes in and among many cortical regions [12]. Thus, gaining a basic understanding of the nature and learning of volitional EEG control may in many cases prove to be a complex and difficult process.

How may we determine which brain modulatory systems are involved in spontaneous and learned control of particular EEG or behavioral/EEG dynamics? A full answer to this question may require invasive experiments (potentially involving patient volunteers who have been implanted with cortical electrodes for clinical purposes), positron emission tomography (PET) experiments that can assess neurotransmitter distributions in the brain, various psychopharmacological manipulations, combined with carefully selected behavioral paradigms, for example those directly manipulating reward levels known to be linked to dopamine release [13]. However, a number of brain modulatory systems may be involved in most state changes and event responses of interest, so this investigation should be expected to be involved.

A possible objection to the model shown in Fig. 1 is that if an adequate BCI function linking the recorded EEG signal to the target behavioral or event/context parameter(s) of interest proves to be linear, then constructing a more elaborate BBI function linking EEG data first to EEG sources, then to their natural modes of spectral modulation, and finally to the estimated event/context or behavioral measure may not give a better-performing estimator. The proposed EEG source modulator model, however, is nonlinear as it operates on source $(\log )$ power spectra. Linear or other functions of the estimated source and source modulator time courses, therefore, involve additional information and might well have advantages over direct (and particularly, linear) BCI estimation. However, use of power spectral estimates ignore source signal phase and with it, precise latency information available in the time- 
domain data. Thus, applying a joint linear (or other) estimator to combine timedomain and time/frequency-domain data could improve performance over a timedomain estimator alone.

Recently Bigdely Shamlo and colleagues demonstrated a successful application of such an approach [14]. We reported a method for estimating the probability that a briefly presented visual image contained a rare target feature - an airplane feature in a stream of satellite ground images presented to the subject at a rate of 12 images per second. Near-real time performance in correctly detecting the presentation of single target-bearing images solely from high-density EEG (by combining source timedomain and source spectral modulator information in a linear estimator) was high, giving an area under the ROC curve of over $90 \%$ for most subjects.

Like most BCI projects, this project did not expressly capture subject behavioral information. However, it did allow use of maximally independent EEG sources capturing potentials induced by characteristic subject eye gaze behavior following target appraisal, unlike BCI systems built to serve completely paralyzed subjects. Although the very rapid serial visual presentation (RSVP) did not reward normal saccadic eye movements, independent components accounting for eye movements following target perception was found to carry some target classification information (though of less value compared to several brain EEG source responses).

The BBI model shown schematically in Fig. 1 does not propose a method for combining EEG and behavioral data, in particular body motion capture data. This is a topic that both requires and deserves much attention and exploration. Of particular interest is to determine the extent to which it is desirable to solve the biomechanical inverse problem, estimating which muscle actions produce the observed sequence of body movements, before estimating links between EEG source activities, body movements, and operator mental state or reactions [8].

Finally, can the proposed MoBI-based BBI systems be practical for widespread application, or must they remain basic research tools? EEG spatial filtering requires the availability of a relatively high number of scalp EEG recording channels. Typically, BCI designers have attempted to maximize signal to noise ratio by restricting the number of channels used in the classifier, an approach that might also lower the cost of the system, if realized using currently available technology. To date, body motion capture (mocap) systems also remain quite expensive. Thus, can the proposed MoBI-based BBI systems become practical for routine application, even in (e.g.) high-value military or civilian environments? Here, the rapid progress of electronic fabrication methods, allow microminiaturized data acquisition and processing units based on flexible thin-film technologies should allow development and relatively low-cost deployment of wearable high-density EEG and behavioral monitoring systems within a few years [15]. Such systems should be readily applicable to some important problems, for example alertness monitoring of shiftwork operators of high-value, high-risk systems [16]. Full realization of the MoBIbased BBI concepts discussed here will likely require a great deal more basic and 
applied research in many laboratories combining expertise in several fields of neuroscience, mathematics, and engineering.

\section{References}

1. Makeig, S., Gramann, K., Jung, T-P., Sejnowski, T.J., Poizner, H.: Linking brain, mind and behavior: The promise of mobile brain/body imaging (MoBI). Int. J. Psychophysiolog. (accepted for publication)

2. Makeig, S., Jung, T-P., Ghahremani, D., Bell, A.J., Sejnowski, T.J.: Blind separation of auditory event-related brain responses into independent components. Proc. Natl. Acad. Sci. USA. 94, 10979-10984 (1997)

3. Makeig, S., Westerfield, M., Jung, T-P., Covington, J., Townsend, J., Sejnowski, T.J., Courchesne, E.: Functionally independent components of the late positive event-related potential during visual spatial attention. J. Neurosci. 19, 2665-2680 (1999)

4. Jung, T-P., Makeig, S., Humphries, C., Lee, T-W., McKeown, M.J., Iragui, V., Sejnowski, T.J.: Removing electroencephalographic artifacts by blind source separation. Psychophysiolog. 37, 163-178 (2000)

5. Jung, T-P., Makeig, S., Westerfield, M., Townsend, J., Courchesne, E., Sejnowski, T.J.: Analysis and visualization of single-trial event-related potentials. Human Brain Mapping $14,166-85$ (2001)

6. Makeig, S., Westerfield, M., Jung, T.P., Townsend, J. Courchesne, E. Sejnowski, T.J.: Dynamic brain sources of visual evoked responses. Science 295, 690-694 (2002)

7. Delorme, A., Makeig, S.: EEGLAB: An open source toolbox for analysis of single-trial EEG dynamics including independent component analysis. J. Neurosci. Meth. 134, 9-21 (2004)

8. Delp, S.L., Anderson, F.C., Arnold, A.S., Loan, P., Habib, A., John, C.T., Guendelman, E., Thelen, D.G.: OpenSim: Open-source software to create and analyze dynamic simulations of movement. IEEE Trans. Biomed. Eng. 54, 1940 - 50 (2007)

9. Makeig, S., Delorme, A., Westerfield, M., Jung, T-P., Townsend, J., Courchesne, E., Sejnowski, T.J.: Electroencephalographic brain dynamics following manually responded visual targets. PLOS Biol. 2, 747-762 (2004)

10. Onton, J., Delorme, A., Makeig, S.: Frontal midline EEG dynamics during working memory. NeuroImage 27, 341-356 (2005)

11. Makeig, S., Enghoff, S., Jung, T-P., Sejnowski, T.J.: A natural basis for efficient brainactuated control. IEEE Trans. Rehab. Eng. 8, 208-211 (2000)

12. Delorme A, Makeig S. EEG changes accompanying learned regulation of $12-\mathrm{Hz}$ EEG activity. IEEE Trans. Neural Sys. Rehab. Eng. 2, 133-136 (2003)

13. Arias-Carrión, O., Pöppel, E.: Dopamine, learning and reward-seeking behavior. Act. Neurobiol. Exp. 67, 481-488 (2007)

14. Bigdely-Shamlo, N., Vankov, A., Ramirez, R.R., Makeig, S.: Brain activity-based image classification from rapid serial visual presentation. IEEE Trans. Neural Syst. Rehabil. Eng. 16, 432-441 (2008)

15. Chin-Teng Lin, C-T., Ko, L-W.; Chiou, J-C., Duann, J-R., Huang, R-S., Liang, S-F., Chiu, T-W., Jung, T-P.: Noninvasive neural prostheses using mobile and wireless EEG. Proc. IEEE 96, 1167-1183 (2008)

16. Makeig, S., Inlow, M.: Lapses in alertness: Coherence of fluctuations in performance and the EEG spectrum. Electroencephalogr. Clin. Neurophysiol. 86, 23-35 (1993) 